\title{
Aspectos interculturales en los libros de texto gratuitos de primaria, en México.
}

\section{Intercultural aspects in free primary textbooks, in Mexico.}

Esta obra está bajo una Licencia Creative Commons Atribución 4.0 Internacional. DOI: $10.32870 /$ sincronia.axxiii.n75.20a19

\section{Martha Vergara Fregoso ${ }^{1}$}

Departamento de Estudios Internacionales / Universidad de Guadalajara

(MÉXICO)

mavederu@yahoo.com.mx

\section{Guillermina Rivera Moreno ${ }^{2}$}

Departamento de Estudios Mesoamericanos y Mexicanos / Universidad de Guadalajara (MÉXICO)

guillerivmor@hotmail.com

\section{José Antonio Méndez Sanz ${ }^{3}$}

Universidad de Oviedo

jatokero@gmail.com

(ESPAÑA)

Recibido: $25 / 10 / 2018$

Revisado: $12 / 11 / 2018$

Aprobado: 06/12/2018

\section{RESUMEN}

\footnotetext{
${ }^{1}$ Doctora en Educación en el Departamento de Estudios Internacionales del Centro Universitario de Ciencias Sociales y Humanidades de la Universidad de Guadalajara. mavederu@yahoo.com.mx

${ }^{2}$ Maestra en Estudios de Filosofía en México. Profesora-Investigadora en el Departamento de Estudios Mesoamericanos y Mexicanos del Centro Universitario de Ciencias Sociales y Humanidades de la Universidad de Guadalajara. guillerivmor@hotmail.com

${ }^{3}$ Doctor en Filosofía en la Universidad de Oviedo, jatokero@gmail.com
} 
El documento contiene los primeros resultados de una investigación enfocada en el análisis del discurso sobre interculturalidad y los valores implicados que se identifican en los libros de textos gratuitos de educación primaria en México ${ }^{4}$, a partir del supuesto de que estos materiales tienen una clara función orientadora, y son los instrumentos básicos que acompañan a los agentes educativos en su práctica docente y en la formación académica y ética del alumno.

El análisis referido se focaliza en la educación intercultural bilingüe que se desarrolla en México, una propuesta nacional que, de manera equivocada, dirige su atención sólo a las comunidades indígenas. Se trata de un concepto que ha cobrado relevancia, ya que aparece en el ámbito pedagógico como una constante cuando se abordan temas para la reflexión y las prácticas educativas, y como propuesta educativa, vinculada al intercambio, al enriquecimiento mutuo, y a la cooperación entre personas y grupos.

El documento contiene cinco apartados; por un lado, se describen los antecedentes y las reformas educativas que se han sucedido en el Sistema Educativo en México; posteriormente, se presenta una discusión sobre conceptos fundamentales de la investigación, como son la interculturalidad y la diversidad; en el tercer apartado se hace mención de la metodología empleada; finalmente se presentan algunos resultados y las conclusiones del estudio.

Palabras clave: Educación intercultural. Diversidad. Libros de Texto Gratuitos. Valores sociopolíticos. Valores culturales.

\section{ABSTRACT}

This document includes the results of a research focused on the discourse analysis on interculturality and values identified in primary school's free textbooks in Mexico, based on the assumption that these materials have a clear guiding function, and that they are basic instruments that accompany educational agents in their teaching practices, and the student's academic and ethical education.

This analysis focuses on bilingual intercultural education developed in Mexico, a national proposal that mistakenly directs its attention only to indigenous communities. It is a concept that has become relevant, since it appears in the pedagogical field as a constant aspect when addressing

${ }^{4}$ Esta investigación está financiada por el Programa 7.1-2018 del Centro Universitario de Ciencias Sociales y Humanidades de la Universidad de Guadalajara. 
issues for reflection and educational practices, and as an educational proposal, linked to exchange, mutual enrichment, and cooperation between people and groups.

This document contains five sections; on the one hand, the background and educational reforms that have taken place in the Mexican Educational System are described; afterwards, a discussion on fundamental concepts of this research, such as interculturality and diversity is presented; in the third section the methodology used for this research is mentioned; and finally some results and conclusions of the study are presented.

Keywords: Intercultural education. Diversity. Free Textbooks. Sociopolitical values. Cultural values.

\section{Introducción}

El tema de este artículo se deriva de la investigación: "Los contenidos discursivos en los libros de texto gratuitos de educación primaria en México", que a su vez forma parte de una investigación interinstitucional e internacional mayor, que lleva por título: "La educación intercultural desde la voz de los agentes educativos" 5 . El trabajo tiene como propósito iniciar el análisis del discurso y los valores relacionados con la interculturalidad, que se encuentran en los libros de texto gratuitos de primaria en México, y observar cómo son tratados y enseñados, en principio.

Como consecuencia de lo anterior, se parte del reconocimiento de la importancia que tiene la diversidad cultural y la educación intercultural en el país, ya que más allá de ser entendidos como aspectos básicos para la formación en valores, se han quedado en el nivel de conceptualización o teorización; por tanto, hasta se podría afirmar que no se ha logrado producir en los alumnos un cambio verdadero de actitudes hacia el otro, y tampoco el conocimiento de la realidad social, a pesar de los esfuerzos que se han hecho en México para construir una educación intercultural.

\section{Algunos antecedentes}

\footnotetext{
${ }^{5}$ Esta investigación es interinstitucional e internacional, financiada por el CONACYT; y se realiza en México, Guatemala, Perú, Venezuela, Chile y Colombia.
} 
El Sistema Educativo Nacional en México, hoy Secretaría de Educación Pública (SEP), ha tenido tres grandes reformas educativas: 1960, 1973 y 1993; la última de ellas trajo consigo una serie de manifestaciones sociales, principalmente de indígenas en la lucha por los derechos humanos, no sólo en México, ocurridas en la década de 1990 de modo particular. Además, se han experimentado otros cambios igualmente significativos ("reformas") basados en las evaluaciones aplicadas al sistema educativo, entre los años 2004 y 2011 y que se hacen visibles en la Reforma Integral de la Educación Básica (RIEB) ${ }^{6}$.

Es decir, han ocurrido modificaciones importantes que hasta hoy se perciben no sólo en el discurso político de México, sino en el educativo: en la formación de maestros, en los servicios, planes y programas educativos y, consecuentemente, en los contenidos de los libros de texto gratuitos.

Los pronunciamientos que acompañan las últimas reformas se dirigen hacia las prácticas educativas, y tienden a promover acciones y "objetivos más humanos", y éticos. El discurso ha acentuado expresiones como: "competencias para la vida", "educación de calidad para todos", "educación para la inclusión" y "educación en valores", entre otras; también ha resaltado la "necesidad de conocimientos y enfoques diferentes en torno a los derechos humanos, el reconocimiento de la diversidad cultural, la heterogeneidad, la tolerancia, el respeto del y por el otro, el reconocimiento de la diversidad, la multiculturalidad y la pluralidad, etc.; en suma, un discurso con un enfoque integral e "intercultural".

Adicionalmente, se pone énfasis en una educación "que responda a las necesidades de desarrollo de México en el siglo XXI [y a la] heterogeneidad étnica, socioeconómica, cultural y de género de los estudiantes, y liderazgo tanto a nivel escolar como comunitario" (Ruiz, 2012: 53, 54). Se advierte entonces que los discursos educativos evidencian cambios para

${ }^{6}$ Reforma Integral de la Educación Básica (Decreto para la educación básica en México: Pre-escolar, Primaria y Secundaria). La RIEB responde a una intención de política educativa expresada tanto en el Plan Nacional de Desarrollo 2007-2012, como en el Programa Sectorial de Educación, correspondiente a esta administración federal (Ruiz, 2012: 52). 
[...] la formación de una ciudadanía [más] democrática, llevando al aula formas de convivencia y de reflexión acordes con los principios y valores de la democracia, [cambios para la atención] adecuada [de] la diversidad cultural y lingüística, estilos de aprendizaje y puntos de partida de los estudiantes, así como relaciones tutoras que valoran la individualidad y potencializan el aprendizaje con sentido (Fortoul, 2014: 47).

Como resultado de lo anterior, surgen temas y proyectos educativos en torno a la "interculturalidad", como una mayor insistencia por educar a los niños y a la sociedad para la interculturalidad, y para el respeto y reconocimiento del otro. De igual manera, se articulan proyectos que incorporan dinámicas enfocadas en la "educación intercultural bilingüe"; y que pregonan una educación de calidad para "todos", o una educación "de calidad" "en valores"; aparecen luego exigencias en la preparación del docente para una práctica de actividades con "nuevos énfasis transversales del currículum, la enseñanza en contextos multiculturales o la atención a otras formas de diversidad en el aula" (Ruiz, 2012: 55).

\section{Marco teórico-conceptual}

En el trabajo de investigación que se realiza, el punto de arranque es el análisis de los conceptos de interculturalidad y diversidad; para este caso, la interculturalidad se entiende como una forma posible de relación: por tanto, si se conoce y reconoce la diversidad cultural, y existe un cierto diálogo entre culturas, se produce el mutuo enriquecimiento.

Es evidente que la sociedad contemporánea ha evolucionado con el tiempo, de esta manera, lo que antes se consideraba como normal, ahora se ha convertido en diverso; esto es el punto de partida para afirmar que la diversidad constituye actualmente la norma, no la situación excepcional. Por tanto, en estos momentos, si la norma es la diversidad, de ella debe emerger cualquier planteamiento o proyecto educativo realista. En este sentido, es imprescindible considerar lo diverso en la estructuración del sistema educativo, si se quiere dar una respuesta apropiada a la población escolar. 
Se parte del supuesto de que la diversidad alude a la riqueza infinita de oportunidades, fuentes, referentes y espacios respecto a temas, problemas y soluciones, por lo que, indudablemente, la diversidad es un elemento omnipresente en el mundo y a la vez impulsor permanente de transformación, innovación y desarrollo. La diversidad cultural supone la diferencia, el diálogo entre lo distinto y el establecimiento de canales recíprocos de comunicación, aprendizaje y experiencias; en tal sentido, la diversidad es fuente de creatividad, originalidad y cambio. Asimismo, se asume que la diversidad cultural supone una fuerte dimensión colectiva que hace que los seres de la Tierra se conciban en un amplio espacio de relaciones, existencias e interacciones. Es por ello que se puede considerar también como el escalón de la individualidad y del reconocimiento de la existencia del igual o del diferente, con proximidad o lejanía, pero con la presunción de formas de potencial coexistencia, bienestar y desarrollo.

Así, lo intercultural aparece en el discurso político y educativo oficial como un concepto complejo de entender y a la vez paradójico, por su carácter moralista y polisémico, como lo conciben algunos teóricos, como Eduardo Restrepo y Günther Dietz, entre otros. Lo anterior, según las investigaciones realizadas por Dietz y Mateos, "remite a la necesidad de estudiar las diferentes respuestas educativas interculturales, multiculturales, bilingües o indigenistas desde una óptica más amplia que la pedagógica" (Dietz y Mateos, 2009:16-17). En opinión de Restrepo, la interculturalidad lleva una

[...] tendencia a la moralización [que] se asume en un registro de lo bueno/malo. Cuando operamos en el registro moral, nos movemos en el campo de las convicciones y principios [valores] que no permiten ninguna sombra de duda: o se está de un lado o del otro, no hay posiciones intermedias ni neutrales (Restrepo, 2014: 24).

Ahora bien, dentro del discurso central de la reforma educativa de los países de Europa occidental y de América Latina, actualmente podemos percibir una educación en valores, con esos dos polos de conciencia moral sobre la interculturalidad de los que habla Restrepo. Se comprende que, en el fondo, tiene un sentido positivo, de lo bueno, por creer que es incluyente de y para las diferencias e 
interactiva; y por el otro lado, porque es claro que no opera acertadamente en la práctica social, como la generalidad esperaría. Lo intercultural se define como

[...] una filosofía que se esfuerza por crear una diversidad cultural, tratando de comprender las diferencias culturales, ayudando a la gente a apreciar y gozar las contribuciones hechas por distintas culturas en sus vidas, así como asegurar la completa participación de cualquier ciudadano para derribar las barreras culturales (Michael y Thompson, 1995; citado en Hidalgo, 2005: 79).

De esa manera, la interculturalidad en educación, implica "la cualidad de crear y sustentar currículas, actividades académicas, programas y proyectos que desarrollen un vivo interés con respecto a todas las culturas humanas" (Acante, 1996; citado en Hidalgo, 2005: 79).

En México, la educación intercultural es una propuesta nacional que, de manera equivocada, dirige su atención sólo a las comunidades indígenas, y afirma que aspira a la formación y el desarrollo de la población en un contexto de equidad y de reconocimiento a la diversidad cultural, por ello se denomina educación intercultural bilingüe y a través de ella se pretende dar respuesta a los requerimientos políticos, filosóficos, culturales y lingüísticos de las diferentes culturas. El planteamiento es interesante porque considera una educación que integra la cultura propia después de siglos de dominación y discriminación, y de la operación de programas asimilacionistas que fomentaron la ruptura de la identidad de los pueblos indígenas y propiciaron el agravamiento de la exclusión y el racismo (Vergara et al., 2008).

El énfasis en lo intercultural nace como una determinación para plantear la educación en contextos multiculturales, lo cual supone reciprocidad y diálogo desde su reconocimiento, valoración y, a la vez, una solidaridad operativa. Se trata de un concepto que ha cobrado un sentido importante, ya que aparece constantemente en el ámbito pedagógico cuando se abordan temas para la reflexión y las prácticas educativas. De tal manera que lo intercultural puede ser entendido de varias formas: como ideal, asociado a movimientos de reforma, de renovación, de mejora de la 
enseñanza, o bien, como propuesta educativa, vinculada al intercambio, al enriquecimiento mutuo, a la cooperación entre personas y grupos.

Uno de los objetivos de la educación intercultural es reformar la escuela para que todos los alumnos alcancen la igualdad educativa; sin embargo, la forma en que se opera dista mucho de lograr lo que se propone, ya que hace falta impulsar programas de desarrollo cultural incluyente a escalas regional, local y comunitaria, con propuestas adecuadas a las necesidades de la comunidad, a fin de enriquecer y ampliar el diálogo entre sus integrantes (Vergara, 2012).

\section{Metodología seguida en el estudio}

La metodología utilizada es de corte cualitativo, porque el objeto de investigación se ubica en el ámbito social, en el que se evidencia la existencia de diversas problemáticas que no se pueden explicar ni comprender en toda su extensión desde la metodología cuantitativa; para ello se ha realizado la revisión y el análisis de la dimensión conceptual de interculturalidad, de las políticas relacionadas con educación intercultural en México, de la Reforma Educativa de las últimas décadas, así como del discurso contenido en los libros de texto gratuitos de primaria.

Es un estudio basado en la técnica de investigación, y en el análisis de contenidos; con ello el interés reside no sólo en la descripción de tales contenidos, sino que, en la medida de ser tratados y analizados, los resultados podrían aportar elementos interesantes para realizar una propuesta temática más viable, orientada a mejorar los principios y prácticas de la educación intercultural en la escuela primaria. La pregunta principal de este proyecto, en principio, se inclinó por investigar, con base en los mensajes de la reforma educativa, cómo son abordados y presentados los aspectos relacionados con la interculturalidad en el contenido de los libros de textos gratuitos de la SEP, incluyendo en esos aspectos lo diverso, los grupos humanos, el respeto y las relaciones con el otro, el conocimiento de la diversidad y de lo multicultural en México, entre otros.

\section{Algunos resultados}


Los libros de texto gratuitos de primaria revisados para el presente estudio fueron principalmente:

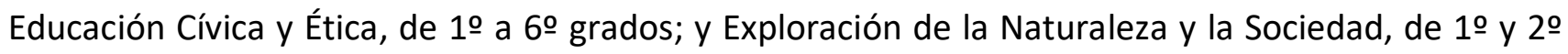
grados; en su introducción, estos libros emiten mensajes significativos del discurso propio del Sistema Educativo Nacional, y esperan, entre otras cosas, que el alumno desarrolle una serie de "competencias básicas para la vida diaria"; se plantea también que "gracias a esta reforma educativa, el libro responda a las demandas de la sociedad mexicana, añadiendo contenido que ayude al alumno a realizar una vida en sociedad más adecuada" (SEP, 2010a: 3).

La investigación da cuenta de los primeros resultados obtenidos tras la revisión de algunos indicadores determinados para el estudio de los libros de texto gratuitos de primaria. Cabe mencionar que se han realizado ciertos cambios en estos materiales; su primera edición se realizó en 2008; hubo una segunda en 2009; y para efectos del presente trabajo se consideraron algunos ejemplares correspondientes a la tercera edición (2010) y otros a la cuarta (2014), distribuidos en los ciclos escolares entre el 2011 y el 2016.

Cada libro por grado y asignatura está dividido en bloques; por ejemplo: en el material del primer año se identificaron los siguientes: "Me conozco y me cuido"; "Me expreso, me responsabilizo y aprendo a decidir"; "Conozco y respeto a las personas que me rodean"; "Construimos reglas para vivir y convivir mejor"; y "Dialogamos para solucionar diferencias y mejorar nuestro entorno". A la vez, cada bloque se subdivide en cinco apartados más, dentro de los cuales hay una introducción al tema [en el apartado Platiquemos], el abordaje del contenido relacionado con algún suceso de la historia [Para aprender más], algunas actividades [Para hacer y juegos y actividades] y, por último, una autoevaluación (SEP, 2010a).

Los indicadores utilizados en esta fase de la investigación ponen atención en manifestaciones y/o valores relacionados con la interculturalidad; este término, per se, encierra una serie de tópicos que igualmente se abordan, tales como: grupos sociales étnicos, nacionales y la diversidad; principios de respeto, humildad, tolerancia, equidad, igualdad, reconocimiento de nosotros y los otros; y principios políticos de legalidad y legitimidad de los diversos grupos, con 
énfasis en la cultura popular, lo rural, la relación con la diversidad y la naturaleza, y las tradiciones y el folklore. ${ }^{7}$

Los libros de texto subrayan un conjunto de valores sociales y culturales en pro del reconocimiento y el respeto por la diversidad; no obstante, es evidente que esa formación se encuentra muy lejos de lo que en la práctica cotidiana escolar sería la interculturalidad; por ejemplo, hasta donde se ha revisado para efectos de esta investigación, no se habla de la realidad social de las comunidades indígenas; más aún: dentro de la misma escuela, ni los maestros ni las autoridades educativas conocen suficientemente la cultura de los migrantes o de los estudiantes indígenas en escuelas generales de la zona urbana.

Ciertamente, se hace referencia a algunos asuntos de las personas diversas, es el caso de los indígenas, pero solamente se destaca la cuestión patrimonial, de pertenencia o de identidad, y no se ofrece información real de su contexto, o de las condiciones de la población actual; algunas evidencias de lo anterior se encuentran en los libros de texto gratuitos (SEP, 2010a):

Como se reconoce en nuestras leyes, México es un país multicultural. Eso significa que en él todavía se conservan, por fortuna, diferentes grupos indígenas, descendientes directos de aquellos que fundaron su grandeza. Se hablan en nuestro país diversas lenguas y en él se vive conforme a diferentes costumbres (51).

El respeto debe tomar en cuenta la diversidad que caracteriza a los seres humanos. Formas parte de una gran nación plulingüe y pluricultural (55).

La diversidad cultural se ve en gran cantidad de fiestas, tradiciones, música, comida, bailes y trajes típicos que caracterizan a cada región de nuestro territorio. [...] la variedad de rasgos culturales es parte de la vida de las mexicanas y los mexicanos, quienes en la vida diaria los hemos hecho perdurar por mucho tiempo, porque los seres humanos defendemos nuestra manera de ser y expresar nuestras tradiciones y nuestra historia (SEP, 2010b: 56).

7 Cabe señalar que gran parte de los indicadores mencionados, fueron tomados de los resultados de distintos seminarios derivados del proyecto de investigación titulado: "Filias y fobias de los niños escolares en Jalisco" realizado en 1995. 
Por otra parte, en el Libro de Texto Gratuito de 20 Grado (SEP, 2010b) aparece la paradoja de la universalización de las leyes y de una democratización igualitaria, acentuando errores históricos que hoy mismo persisten, como la dificultad de asegurar la convivencia real con la diversidad:

La vida en sociedad se facilita cuando quienes la forman se ponen de acuerdo sobre las normas que seguirán todos (73).

En la democracia, que es la forma de vida que en México queremos tener, el respeto a las normas ayuda a lograr la convivencia respetuosa, justa, igualitaria y solidaria (74).

Hay algo que llama la atención en los libros de texto (SEP, 2010b), como parte del mensaje de igualdad, equidad y respeto por la diversidad: un discurso que, si se materializara en la práctica, se convertiría en el ideal de los pueblos indígenas, ya que significaría reconocer sus espacios de participación para impulsar su desarrollo en los ámbitos económico, político y educativo, contribuyendo así a la construcción social nacional.

La participación implica acción conjunta y tender puentes entre distintos proyectos, gustos, maneras y razones de hacer las cosas (92).

Aprende a participar y a tomar con los demás decisiones justas y apegadas a la legalidad. Ésta es la clave para vivir con armonía, bienestar y democracia (97).

Por distintas que te sean tales personas, ellas componen el grupo al cual perteneces (50).

Quienes hemos tenido mayores oportunidades, estamos más obligados a lograr que haya en México condiciones de equidad y justicia. En este tema tú, estudiante, puedes hacer algo. Y puedes hacerlo desde ahora. Por ejemplo, teniendo mayor respeto por la cultura, la vestimenta, la lengua y las creencias de los diferentes pueblos indígenas, que deben ser nuestro orgullo, porque forman parte de la riqueza y la diversidad cultural que nos distingue en el mundo (39).

Formas parte de una gran nación plurilingüe y pluricultural, es decir, las personas pueden hablar distintas lenguas y tener diferentes costumbres y tradiciones (55). 
La diversidad cultural se ve en gran cantidad de fiestas, tradiciones, música, comida, bailes y trajes típicos que caracterizan a cada región de nuestro territorio (56).

Un elemento importante que distingue a los pueblos del mundo y les da identidad es la lengua con la que se comunican (58).

Vivir en sociedad implica compartir tiempo, lugares y bienes o cosas (SEP, 2010b: 92).

Respecto a los valores más recurrentes identificados en los libros de texto, se puede decir que, por tratarse de la asignatura relacionada con ética y formación cívica, el discurso en torno a la interculturalidad está presente y se ha identificado una importante gama de valores o indicadores de carácter sociocultural, y sociopolítico, los cuales remarcan una nueva fase significativa del discurso oficial hacia la defensa de los derechos humanos. Entre los temas más citados en los contenidos se encuentran los siguientes: respeto, reconocimiento de nosotros y los otros, derechos humanos, libertad, tolerancia, igualdad, equidad, principios de legalidad, principios de democracia, reciprocidad, justicia y honestidad.

Entre los valores menos citados están: confianza en las instituciones, prudencia, solidaridad, relación con la diversidad y la naturaleza, cultura popular, tradición y folklore (en ese orden).

Es un hecho que la educación intercultural se está dando actualmente, sobre todo en el nivel básico, con el fin de fortalecer valores de manera transversal, entrecruzándolos en las distintas asignaturas sociales y de las humanidades. Al respecto, en opinión de José Domínguez:

Los valores son creaciones humanas y construcciones sociales dinámicas, evolutivas e indefinidamente perfectibles, que se trasmiten y asimilan por aprendizaje social. Los valores son fruto de la reflexión colectiva de los grupos humanos y de la acción comunicativa sobre los modos de satisfacer las necesidades humanas naturales $\mathrm{y}$ culturales y de mejorar las situaciones personales y sociales (2014: 4).

Pero para el autor también tienen su bipolaridad [como ya lo había hecho notar de alguna manera Restrepo], es decir, tienen un lado positivo y un lado negativo: "Todo lo que consideramos 
favorable y conducente a las situaciones deseables y satisfactorias lo calificamos como valor. Todo lo que consideramos desfavorable y contrario a las situaciones deseables y satisfactorias lo calificamos como contravalor" (Domínguez, 2014: 4). En esas dos formas hallamos calificaciones de lo bueno y de lo malo, y por consiguiente, prejuicios.

La función de la educación como preservadora, estabilizadora y controladora de la situación social existente, permite transmitir, conservar, promover y consolidar los patrones de conducta, las ideas y valores socialmente aceptados, creándose en este proceso una cualidad superior, traducida en nuevos valores, para la interpretación de las nuevas realidades que construye cada generación.

En el discurso educativo y la educación intercultural en México, se puede observar cierta incongruencia en los contenidos de los libros de texto, ya que, a pesar del énfasis en la formación de valores que se establece como política nacional, así como el respeto a las diferencias (físicas, culturales, sociales, religiosas, etc.) de las personas que habitan el mundo y México, la interculturalidad no se percibe en los contenidos de los libros de texto tal como ha sido conceptualizada teóricamente por los autores tratados y como se propone en la práctica cotidiana. Habría que utilizar estrategias didácticas para acercar más a los niños a las realidades y problemas sociales y culturales que viven los diferentes grupos; por ejemplo, la situación de los indígenas en desventaja, la pobreza, el bullying escolar, las prácticas de exclusión y racismo en la escuela y en la casa, etc.

Es importante mencionar que los problemas de violencia y discriminación que hay en las escuelas de México cada vez aumentan más. Este tipo de conflictos no se resuelve entre los propios niños por la falta de respeto, de aceptación o tolerancia del otro; por sus diferencias, por sus fobias, y otras razones de carácter no únicamente social, sino psicológico. Dentro del contenido de los libros de texto, aunque se insiste en el discurso por la empatía con el otro, o en el respeto por la diversidad de personas, culturas, razas, personalidades, edades, condiciones físicas de las personas, etc., sigue persistiendo una violencia cercana al racismo. El bullying en las escuelas es un típico ejemplo de intolerancia y de fobia hacia el otro, que, si bien se deriva, entre muchos factores, de la 
violencia intrafamiliar, también es un síntoma de la patología social y humana, y de las formas de rechazo hacia el otro, hacia lo diferente.

De acuerdo con los autores Pacheco, Navarro y Cayeros, "Los libros de texto gratuitos (LTG) forman parte de los dispositivos culturales desplegados por el Estado mexicano para lograr la homogeneización de la población dentro de la concepción: una patria, una nación" (Pacheco, Navarro, Cayeros, 2011: 1).

En los libros de texto (SEP, 2010a) hay una marcada presencia del discurso por el respeto hacia la persona:

Respeta a todas las personas; es decir, toma en cuenta su manera de ser, edad y sus derechos (14).

Respetar a una persona significa tomar en cuenta su edad, su necesidad de protección, sus características físicas y su manera de ser. Si es de corta edad, respetarla es ayudarla a aprender, a crecer, a convivir con los demás y desarrollarse con salud y alegría. Si es persona mayor tendrá necesidades diferentes (21).

La actitud de respeto y la capacidad de empatía te harán saber que todas las personas deben ser consideradas seres valiosos que es necesario proteger (33).

En los contenidos de los libros revisados se observan múltiples mensajes relativos a la diversidad y los diferentes grupos, también en torno al respeto y a la protección de los mismos, y a pesar de existir ejercicios prácticos en los planes didácticos, es necesario enseñar sobre la base de un conocimiento tangible de la realidad social dentro y fuera de las escuelas, y de las personas diferentes.

\section{Conclusiones}

La educación intercultural debería considerar una serie de aspectos vinculados a las culturas humanas, a la diversidad cultural, a las diferencias culturales, a la otredad, al multiculturalismo, a las culturas indígenas, a lo autóctono o a las minorías autóctonas, al discurso multicultural, a la 
educación bilingüe intercultural, a los valores moralizantes alrededor del término intercultural (culturales, sociales y políticos), a la identidad, a lo heterogéneo, a lo diferente, a lo comunal, a una interacción y relación entre dos, a la multiplicidad, y a las relaciones económicas, culturales y sociales, entre otros puntos.

Los libros de texto gratuitos y comerciales (de nivel primaria para el caso de esta investigación), tienen una clara función y son los instrumentos básicos que acompañan a los agentes educativos en su tarea docente y en la formación académica del alumno; significativamente forman y orientan en el manejo de competencias y habilidades educativas, pero también aportan conocimientos, en ocasiones sesgados, que ideologizan con base en filosofías o maneras de pensar sobre diversos aspectos del entorno social, histórico y político; por tanto, el análisis de sus contenidos podría acercarnos a identificar problemáticas sociales y educativas vistas y no vistas en los programas didácticos, y a vislumbrar el potencial impacto en la sociedad del conocimiento; de ahí la importancia de este estudio, que ha planteado la necesidad de revisar los contenidos escolares en los libros de texto gratuitos de primaria, con el fin de conocer las formas y la problemática en la educación para la diversidad e interculturalidad, desde el desempeño de los actores educativos y la inclinación de los discursos.

Se percibe que hay exclusión a pesar de los esfuerzos realizados; que no existen las mismas oportunidades ni relaciones para todos; y que la educación de calidad, inclusiva e intercultural que tanto se persigue debe ser entendida con más objetividad, con el fin de pasar del discurso a la práctica social. La educación en valores en y para la diversidad y multiculturalidad debe ser comprendida, enseñada y transmitida a la sociedad y a los niños desde los contenidos y discursos de los libros de texto, partiendo de otros enfoques y mediante diferentes prácticas. Tal formación debe trascender más allá de la conceptualización, y el Estado y las instituciones educativas deben repensarla y analizarla mejor para actuar con más certeza.

Valores como la diversidad, el respeto y el reconocimiento del otro se desarrollan en los niños a través del discurso presente en los libros de texto, pero sigue habiendo limitaciones de 
atención y conocimiento educativo intercultural, y actitudes negativas en la práctica social. Aún hay temáticas ausentes en los contenidos de los libros de texto gratuitos, especialmente aquellas que reflejan la realidad social y el conocimiento real de grupos vulnerables, diversos, multiculturales, 0 que resalten actitudes de reconocimiento al otro, al diferente.

\section{Referencias}

Calvo, T.; Ávila, R. (Coords.) (1995) Proyecto de investigación: “Filias y fobias de los niños escolares en Jalisco". Documento de trabajo.

Dietz, G. y Mateos, L. S. (2011). Interculturalidad y educación intercultural en México. Un análisis de los discursos nacionales e internacionales en su impacto en los modelos educativos mexicanos. México: Secretaría de Educación Pública (SEP), Coordinación General de Educación Intercultural y Bilingüe (CGEIB).

Domínguez, J. (junio de 2014). "Educación en valores". Texto en PDF consultado el 25 de julio de 2016,

http://www.caesasociacion.org/educacion menores excluidos/ficheros/educacion en valo res.pdf

Fortoul, B. (2014). "La reforma integral de la educación básica y la formación de maestros", en Perfiles Educativos, vol. 36, núm. 143, México, enero de 2014. Versión electrónica, consultada en julio de 2016: http://www.scielo.org.mx/scielo.php?script=sci_arttext\&pid=S0185-26982014000100021

Hidalgo, V. (2005). “Cultura, multiculturalidad, interculturalidad y transculturalidad. Evolución de un término". Texto en PDF, consultado en julio de 2015, en: http://www.fecchiapas.com.mx/sistema/biblioteca digital/cultura-multiculturalidad-interculturalidad-ytransculturalidad.pdf

Morales, R. [SEP, SEB] (24/II/ 2012). Entrevista "Los programas estratégicos en educación indígena". [Archivo de Vídeo]. Recuperado de: https://www.youtube.com/watch?v=8xriEpJ-014 
Pacheco, L. C.; Navarro, M.; Cayeros, L I. (2011). "Pueblos indios en los libros de texto gratuitos", en Revista Mexicana de Investigación Educativa, vol. 16, núm. 49 (abril- junio de 2011), pp. 525544. Texto en PDF consultado en junio de 2015, en: http://www.scielo.org.mx/scielo.php?script=sci arttext\&pid=S1405-66662011000200009

Restrepo, E. (2013). “Interculturalidad en cuestión: cerramientos y potencialidades” (Artículo en PDF basado en la ponencia preparada para el Primer Seminario Internacional orientado a la discusión de enfoques y perspectivas antropológicas de las relaciones interculturales en el marco del Centro Interdisciplinario de Estudios Interculturales de Indígenas (ICIIS). Santiago de Chile, 21 y 22 de agosto de 2013).

Ruiz, G. (2012). “La Reforma Integral de la Educación Básica en México (RIEB) en la educación primaria: desafíos para la formación docente", en Revista Electrónica Interuniversitaria de Formación del Profesorado, vol. 15, núm. 1, 2012, pp. 51-60. Asociación Universitaria de Formación del Profesorado Zaragoza, España. Consultada en Diciembre de 2015, en: http://www.redalyc.org/pdf/2170/217024398004.pdf

SEP (2010a). Formación Cívica y Ética. Primer grado [FCyE 1]. 3a. ed. México: SEP [reimpr.2011]. SEP (2010b). Formación Cívica y Ética. Segundo grado [FCyE 2]. 3a. ed. México: SEP [reimpr.2011]. SEP (2014). Formación Cívica y Ética. Tercer grado [FCyE 3]. 4a ed. rev. México: SEP [reimpr.2015]. SEP (2014). Formación Cívica y Ética. Cuarto grado [FCyE 4]. 4ạ ed. rev. México: SEP [reimpr.2015] SEP (2014). Formación Cívica y Ética. Sexto. grado [FCyE 6]. 4ạ ed. rev. México: SEP [reimpr.2015]. SEP (2014). Exploración de la naturaleza y la sociedad. Primer Grado [ENS 1]. 4a ed. rev. México: SEP [reimpr. 2015].

SEP (2014). Exploración de la naturaleza y la sociedad. Segundo Grado [ENS 2]. 4ạ ed. rev. México: SEP [reimpr. 2015].

SEP, CONALITEG (Comisión Nacional de Libros de Texto Gratuitos) (2009). Consultado en Septiembre de 2015, en: http://www.conaliteg.gob.mx/index.php/inicio/historia\# 
Vergara, M.; Bernache, G.; Esparza, I. y Cortés, M. (2008). Educación intercultural. Un estudio en las comunidades indígenas en Jalisco. México: SEP; CONACYT, Gobierno del Estado de Jalisco.

Vergara, M. (2012). "La educación intercultural en México. Un reto para la escuela”, Pensar la educación para Iberoamérica. Colombia: Universidad Santo Tomás (USTA). 